\title{
Hybrid PET-dynamic CECT in the management of breast cancer
}

\author{
Rakesh Kumar • Chandan Jyoti Das
}

Published online: 20 January 2009

(C) Springer-Verlag 2009

Breast cancer is the most common cancer and second commonest cause of cancer death after lung and bronchial cancer in women. In Europe, 429,900 cases of breast cancer were diagnosed in 2006 and 131,900 women died from breast cancer in the same year [1]. It has been estimated that 182,460 new cases of breast cancer will have been diagnosed and 71,030 patients will have died from breast cancer in United States in 2008 [2]. Early diagnosis and reliable imaging assessment of response to treatment are essential in the clinical management of breast cancer. Conventional imaging modalities such as screen-film mammography, digital mammography, ultrasonography (USG), and magnetic resonance imaging (MRI), provide only morphological details and do not provide information on the metabolic status of a lesion, which is very important in differentiating a benign from a malignant lesion, and therefore conventional imaging modalities lack specificity.

Since its introduction, positron emission tomography (PET) has been widely shown to be highly useful for the diagnosis of palpable masses, for staging, for obtaining long-term prognostic information, and for demonstrating tumour response to chemotherapy at an early phase or after completion of treatment in patients with breast cancer.

This Editorial commentary refers to the article http://dx.doi.org/ 10.1007/s00259-008-0948-1.

\section{R. Kumar $(\bowtie)$}

Department of Nuclear Medicine,

All India Institute of Medical Sciences,

New Delhi 110029, India

e-mail: rkphulia@yahoo.com

\section{J. Das}

Department of Radiodiagnosis,

All India Institute of Medical Sciences,

New Delhi, India
However, PET is not a very useful modality for screening and early diagnosis of breast carcinoma. The sensitivity of PET is $92 \%$ for lesions $>20 \mathrm{~mm}$, but is only $53 \%$ for primary lesions $<5 \mathrm{~mm}$ [3]. This poor detection of small lesions is due to partial volume effects and low FDG uptake in the tumour as compared to the background breast tissue [4]. A possible solution to this problem is dual time-point acquisition which can improve the diagnostic utility of PET in the detection of breast cancer [5]. FDG uptake in dense breast is high as compared to uptake in the fatty breast, which also hampers the detection of small tumours in dense breast $[6,7]$. Moreover, the poor anatomical resolution of PET further worsens the visibility of small tumours. We have previously systematically evaluated various clinicopathological factors that predict false-negative FDG-PET results in patients with primary breast cancer [8]. We demonstrated that smaller tumour size $(<10 \mathrm{~mm})$ and lower histological grade of the tumour are strong predictors of false-negative FDG-PET results.

With the advances in technology, there is progressive improvement in the spatial resolution of PET. The spatial resolution has been further enhanced with the introduction of the hybrid PET/CT scanner where the PET part of the scanner is equipped with a high-resolution state-of-the-art multidetector CT (MDCT) system. The introduction of this hybrid $\mathrm{PET} / \mathrm{CT}$ scanner has led to a paradigm shift in oncological imaging, and it can be used as a "one-stop shop" not only to diagnose the involvement of various organs but also potentially to characterize the lesions. It is well established that combined PET/CT has higher sensitivity and specificity than PET and CT used separately. The process of malignant transformation is very complex, involving many alterations in the metabolic and functional status of tumour cells. PET and PET/CT heavily rely on the metabolic activity of FDG detected by its PET component. The perfusion component of 
CT using intravenous contrast material is largely unexplored. Perfusion imaging is a functional study which can quantify tumour angiogenesis, blood perfusion, and vascular permeability [9]. It can also evaluate the early effects of therapeutic interventions in malignant solid tumours.

The availability of rapid imaging with MDCT systems and robust computing software has made CT perfusion imaging a reality. Dynamic contrast-enhanced CT (DCECT) can measure the regional blood flow and volume along with mean transit time of blood through the capillaries. Therefore combined, FDG PET/DCE-CT can assess not only glucose metabolism of tumours but also tumour vascularity. Both of these parameters can be used in the differentiation between malignant and benign tumour, in the evaluation of tumour aggressiveness, tumour response to therapy and occult residual tumour, and in the delineation of the tumour during radiotherapy planning. Thus FDG PET/DCE-CT can improve the diagnosis, prognosis, treatment selection and therapy monitoring of various cancers [10].

In a recently concluded study by Akashi-Tanaka et al. found that all breast cancers could be distinguished from normal mammary glands based on the perfusion value which was measured using a 256-row MDCT scanner [11]. The extent of cancer depicted on perfusion images showed excellent agreement with the pathology findings for invasive ductal carcinoma and ductal carcinoma in situ. The results of this study suggest that volume perfusion imaging may be useful for depicting the extent of breast cancer, although further study is needed to determine its clinical relevance. Published literature suggests an increasing role of CT perfusion in the evaluation of solitary pulmonary nodules, colorectal carcinoma, hepatocellular carcinoma and nasopharyngeal carcinoma. DCE-CT imaging has shown that CT perfusion data closely correlates with tumour angiogenesis and reflects microvessel density measurement and vascular endothelial growth factor expression in solitary pulmonary nodules [12]. In a study by Goh et al., the tumour blood flow differed significantly between disease-free and metastatic patients with colorectal cancer [13]. Accurate and precise measurement of hepatic arterial blood flow is possible with DCE-CT. In cirrhotic patients with hepatocellular carcinoma, where PET has a limited role, CT perfusion data from FDG PET/DCE-CT can play an important role in providing quantitative information about tumour-related angiogenesis [14]. Zhou et al. reported that perfusion parameters (blood flow, blood volume), mean transit time and permeability surface) measured with perfusion CT are significantly altered in nasopharyngeal cancer [15].

Metabolic flow relationships of tumours have been demonstrated using different PET tracers [16-18]. However, $\mathrm{H}_{2}{ }^{15} \mathrm{O}$ is not easily available and is not routinely used in clinical practice. In addition, it requires an on-site cyclotron. Mankoff et al. demonstrated the value of combining
FDG metabolic PET data with perfusion data obtained by $\mathrm{H}_{2}{ }^{15} \mathrm{O}$ in locally advanced breast cancer over the course of neoadjuvant chemotherapy [16]. The authors concluded that tumour metabolic and perfusion changes in locally advanced breast cancer can differentiate responders and nonresponders. Patients who had no significant decline in tumour perfusion after 2 months of therapy had poorer disease-free and overall survival. In another study, the same group compared tumour blood flow and glucose metabolism with clinical and pathological parameters and with response to chemotherapy in patients with locally advanced breast cancer [17]. The tumour blood flow and glucose metabolism were significantly higher in tumour than in normal breast. In another study, Hentschel et al. also found significantly higher perfusion and glucose metabolism in tumours than in normal breast tissue [18]. Both these studies showed a positive correlation between glucose metabolism detected by FDG and tumour blood flow.

In a recently published study, Groves et al. found a good correlation between metabolic components of FDG-PET and blood flow as measured by DCE-CT in the evaluation of primary breast cancer [19]. The authors found a significant correlation with SUV when perfusion was normalized to cardiac output. The perfusion and metabolic components of the hybrid PET/CT scanner may be complementary to each other, as shown by Groves et al., and further study is required to standardize the technique and to establish its validity. The importance of tissue perfusion and metabolic coupling is also evident in lung cancer and head and neck squamous cell carcinomas, and may provide additional diagnostic information in patients undergoing PET/CT studies [20, 21]. With functional imaging set to heavily supplant, if not completely replace, many traditional biochemical and physiological tests in the near future, the scope of imaging is bound to expand into new spheres of influence. In keeping with this realistic anticipation, this combined technique of PET and DCE-CT may open up a new horizon in breast cancer research.

Dynamic MRI has been commonly used to measure perfusion of various tumours with implications in the diagnosis, staging and evaluation of treatment response of breast cancer [22]. Semple et al. investigated the relationship between vascular and metabolic characteristics of breast tumour using contrast-enhanced dynamic MRI and FDG PET imaging [23]. The authors found a good correlation between tumour vascularity and metabolism. In another study, comparing dynamic MRI and FDG PET, the results concerning the diagnosis of primary breast lesions were almost identical [24]. As compared to DCECT, dynamic MRI has certain advantages. There is no reaction to intravenous contrast material in MRI, and no radiation exposure to the patient. In the era of rapidly changing technology very soon PET-MRI may be available 
for routine clinical use. Then it will be interesting to see what the role of DCE-CT will be.

Tumour perfusion data obtained by FDG PET/DCE-CT will have certain limitations as the software calculating these data is based on certain assumptions. The perfusion parameter result from time-contrast enhancement curves within an arterial input is uniform. However, contrast enhancement within tumour tissue is nonuniform. Moreover, contrast used for this technique is not ideal to measure correct perfusion and is certainly inferior to ${ }^{15} \mathrm{O}$ perfusion data. The role in hypoxic tumour cells is also uncertain. As do other tests, this technique will also give false-negative and false-positive results under certain conditions. It is known that slowly growing and well-differentiated histological subtypes of tumours such as tubular carcinoma and in-situ carcinoma, and lobular carcinomas, show nearnormal or a mild increase in perfusion. Therefore, falsenegative results will be noted in such tumours. Similarly, false-positive results are likely to occur in patients with a history of recent surgery, chemotherapy and radiotherapy, as such conditions will also lead to increased perfusion.

In conclusion, combined FDG PET/DCE-CT imaging techniques are still evolving and methods of image analysis remain variable and nonstandard. The perfusion data obtained by DCE-CT appear to be promising and likely to have some incremental value over structural and metabolic data provided by PET-CT. The results of initial published studies using perfusion data are encouraging. However, further studies recruiting larger numbers of patients would define the role of the FDG PET/DCE-CT technique and its indications in the management of various cancers in the near future.

\section{References}

1. Ferlay J, Autier P, Boniol M, Heanue M, Colombet M, Boyle P. Estimates of the cancer incidence and mortality in Europe in 2006. Ann Oncol 2007;18:581-92.

2. Jemal A, Siegel R, Ward E, Hao Y, Xu J, Murray T, et al. Cancer statistics 2008 CA. Cancer J Clin 2008;58:71-96.

3. Cermik TF, Mavi A, Basu S, Alavi A. Impact of FDG PET on the preoperative staging of newly diagnosed breast cancer. Eur J Nucl Med Mol Imaging 2008;35:475-83.

4. Belohlavek O. What is the role of FDG-PET in the initial staging of breast cancer. Eur J Nucl Med Mol Imaging 2008;35:472-4.

5. Basu S, Mavi A, Cermik T, Houseni M, Alavi A. Implications of standardized uptake value measurements of the primary lesions in proven cases of breast carcinoma with different degree of disease burden at diagnosis: does 2-deoxy-2-[F-18]fluoro-D-glucosepositron emission tomography predict tumor biology? Mol Imaging Biol 2008;10:62-6.

6. Kumar R, Loving V, Chauhan A, Zhuang H, Alavi A. Dual time point imaging and its potential to improve breast cancer diagnosis with F18-fluorodeoxyglucose positron emission tomography. J Nucl Med 2005;46:1819-24.

7. Kumar R, Chauhan A, Zhuang H, Chandra P, Schnall M, et al. Standardized uptake values of normal breast tissue with 2-deoxy-
2-[F-18]fluoro-D-glucose positron emission tomography: variations with age, breast density, and menopausal status. Mol Imaging Biol 2006;8:355-62.

8. Kumar R, Chauhan A, Zhuang H, Chandra P, Alavi A. Clinicopathologic factors associated with false negative FDG-PET in primary breast cancer. Breast Cancer Res Treat 2006;98:267-74.

9. Zhang J, Wang R, Lou H, Zou Y, Zhang M. Functional computed tomographic quantification of angiogenesis in rabbit VX2 softtissue tumour before and after interventional therapy. J Comput Assist Tomogr 2008;32:697-705.

10. Miles KA. Perfusion imaging with computed tomography: brain and beyond. Eur Radiol 2006;16(Supp1 7):M37-M43.

11. Akashi-Tanaka S, Shien T, Tsukagoshi S, Funabasama S, Miyagawa $\mathrm{K}$, Terada K, et al. Whole-breast volume perfusion images using 256row multislice computed tomography: visualization of lesions with ductal spread. Breast Cancer 2009;16:62-7.

12. Ma SH, Le HB, Jia BH, Wang ZX, Xiao ZW, Cheng XL, et al. Peripheral pulmonary nodules: relationship between multi-slice spiral CT perfusion imaging and tumour angiogenesis and VEGF expression. BMC Cancer 2008;8:186.

13. Goh V, Halligan S, Wellsted DM, Bartram CI. Can perfusion CT assessment of primary colorectal adenocarcinoma blood flow at staging predict for subsequent metastatic disease? A pilot study. Eur Radiol 2008. doi:10.1007/s00330-008-1128-1.

14. Ippolito D, Sironi S, Pozzi M, Antolini L, Ratti L, Alberzoni C, et al. Hepatocellular carcinoma in cirrhotic liver disease: functional computed tomography with perfusion imaging in the assessment of tumour vascularization. Acad Radiol 2008;15:919-27.

15. Zhou Z, Wu J, Han M, Liu R, Chen Z, Huang H, et al. MSCT perfusion analysis of nasopharyngeal cancer. Lin Chung Er Bi Yan Hou Tou Jing Wai Ke Za Zhi 2008;22:150-2.

16. Mankoff DA, Dunnwald LK, Gralow JR, Ellis GK, Schubert EK, Tseng J, et al. Changes in blood flow and metabolism in locally advanced breast cancer treated with neoadjuvant chemotherapy. J Nucl Med 2003;44:1806-14.

17. Mankoff DA, Dunnwald LK, Gralow JR, Ellis GK, Charlop A, Lawton TJ, et al. Blood flow and metabolism in locally advanced breast cancer: relationship to response to therapy. J Nucl Med 2002;43:500-9.

18. Hentschel M, Paulus T, Mix M, Moser E, Nitzsche EU, Brink I. Analysis of blood flow and glucose metabolism in mammary carcinomas and normal breast: a H2(15)O PET and 18F-FDG PET study. Nucl Med Commun 2007;28:789-97.

19. Groves AM, Wishart GC, Shastry M, Moyle P, Iddles S, Britton P, et al. Metabolic-flow relationships in primary breast cancer: feasibility of combined PET/dynamic contrast-enhanced CT. Eur J Nucl Med Mol Imaging 2008. doi:10.1007/s00259-008-0948-1.

20. Miles KA, Griffiths MR, Keith CJ. Blood flow-metabolic relationships are dependent on tumour size in non-small cell lung cancer: a study using quantitative contrast-enhanced computer tomography and positron emission tomography. Eur J Nucl Med Mol Imaging 2006;33:22-8.

21. Bisdas S, Spicer K, Rumboldt Z. Whole-tumour perfusion CT parameters and glucose metabolism measurements in head and neck squamous cell carcinomas: a pilot study using combined positronemission tomography/CT imaging. AJNR Am J Neuroradiol 2008;29:1376-81.

22. Hayes C, Padhani AR, Leach MO. Assessing changes in tumour vascular function using dynamic contrast-enhanced magnetic resonance imaging. NMR Biomed 2002;15:154-63.

23. Semple SI, Gilbert FJ, Redpath TW, Staff RT, Ahearn TS, Welch AE, et al. The relationship between vascular and metabolic characteristics of primary breast tumours. Eur Radiol 2004;14:2038-45.

24. Brix G, Henze M, Knopp MV, Lucht R, Doll J, Junkermann H, et al. Comparison of pharmacokinetic MRI and [18F] fluorodeoxyglucose PET in the diagnosis of breast cancer: initial experience. Eur Radiol 2001;11:2058-70. 